\title{
AN EXAMPLE OF A DOUBLY CONNECTED DOMAIN WHICH ADMITS A QUADRATURE IDENTITY
}

\author{
A. L. LEVIN
}

\begin{abstract}
In this paper we construct a doubly connected domain $D \ni 0$ such that $\iint_{D} f(z) d \sigma=A f(0)+B f^{\prime}(0)$ for any analytic and area integrable in $D$ function $f$, which has a single-valued integral in $D$.
\end{abstract}

1. Introduction. We first introduce the notation (see [1]). Let $D$ be a bounded plane domain. By $L_{a}^{1}(D)$ we denote the set of single-valued analytic functions in $D$ which are integrable in $D$ with respect to the areal measure $d \sigma$, and by $L_{a, s}^{1}(D)$ the subset of $L_{a}^{1}(D)$ consisting of functions with single-valued integral. We say that $D$ admits a quadrature identity (q.i.) relative to $L_{a}^{1}(D)$ (or $L_{a, s}^{1}(D)$ ) if there exist a point $z_{0} \in D$ and complex numbers $A, B$ such that

$$
\int_{D} f d \sigma=A f^{\prime}\left(z_{0}\right)+B f\left(z_{0}\right)
$$

for every $f \in L_{a}^{1}(D)$ (or $f \in L_{a, s}^{1}(D)$ ).

For a discussion of the background of this problem, see [1]. We note only that for a one point q.i., namely

$$
\int_{D} f d \sigma=A f\left(z_{0}\right)
$$

there is no difference between $L_{a}^{1}$ and $L_{a, s}^{1}$. It can be shown [1, Theorem 7] that the validity of $(* *)$ for every $f \in L_{a, s}^{1}(D)$ implies that $D$ is simply connected and, hence, a disc centered at $z_{0}$.

In the present paper we show that the validity of $(*)$ for all $f$ in $L_{a, s}^{1}(D)$ does not imply that $D$ is a simply connected domain. We prove the following

THEOREM. There exists a bounded doubly connected domain $D$ which admits a quadrature identity $(*)$ for all $f \in L_{a, s}^{1}(D)$.

REMARKS. 1. It turns out that the validity of $(*)$ for all $f \in L_{a}^{1}(D)$ does imply that $D$ is simply connected. This fact was proved by $D$. Aharonov and H. Shapiro [1, Theorem 4]. Such a domain $D$ can be found explicitly.

2. Our theorem is closely related to a certain minimal-area problem considered in [2]. In fact, our example shows that the method in [2], as it stands now, is not sufficiently strong to conclude that a certain domain $D$ is

Received by the editors January 5, 1976.

AMS (MOS) subject classifications (1970). Primary 30A80, 30A88. 
simply connected. Such a conclusion would lead to the complete solution of the above-mentioned extremal problem.

3. A recent result (unpublished) of B. Gustafsson shows that if we consider a q.i. of higher order, then for every positive integer $n$ there exists a domain of connectivity $n$ which admits a q.i. of some (unknown) order.

2. We start with some preliminary results.

LEMma 1. Let $D$ be a bounded doubly connected domain. Let its boundary be $C=\Gamma_{1} \cup \Gamma_{2}$, where $\Gamma_{1}$ and $\Gamma_{2}$ are nonintersecting rectifiable Jordan curves, $\Gamma_{2}$ surrounding $\Gamma_{1}$. Then the rational functions from $L_{a, s}^{1}(D)$ are dense in $L_{a, s}^{1}(D)$ in the $L^{1}(D)$-metric.

Proof. Let $\zeta$ be some fixed point in the interior of $\Gamma_{1}$. It is known ${ }^{1}$ that under our topological requirement on $\partial D$ it is possible to approximate (in the $L^{1}(D)$-metric) every function $f \in L_{a}^{1}(D)$ by rational functions with poles at $\zeta$ and at infinity. Let $R_{n}(z)$ be a sequence of such rational functions for a given $f$, i.e. $\int_{D}\left|f(z)-R_{n}(z)\right| d \sigma \rightarrow 0$ as $n \rightarrow \infty$. It is known (see [4, p. 109]) that this condition implies the uniform convergence of $R_{n}(z)$ to $f(z)$ on any closed set interior to $D$. Consequently, we have for some fixed path $\gamma \subset D$ which surrounds $\Gamma_{1}$ :

$$
\left|\int_{\gamma} R_{n}(z) d z\right|=\left|\int_{\gamma}\left(R_{n}(z)-f(z)\right) d z\right| \leqslant c_{\gamma} \max _{\gamma}\left|f(z)-R_{n}(z)\right| \rightarrow 0 .
$$

This means that the residue $\alpha_{n}$ of $R_{n}(z)$ at point $\zeta$ tends to zero. Let $\tilde{R}_{n}(z)=R_{n}(z)-\alpha_{n} /(z-\zeta)$; then $\tilde{R}_{n}(z) \in L_{a, s}^{1}(D)$ and we have

$$
\int_{D}\left|f-\tilde{R}_{n}\right| d \sigma \leqslant \int_{D}\left|f-R_{n}\right| d \sigma+\left|\alpha_{n}\right| \int_{D} \frac{d \sigma}{|z-\zeta|} \rightarrow 0
$$

LEMMA 2. Let $D$ be as in Lemma 1 and contain zero, and let $f(z)$ be continuous in $\bar{D}$ and analytic in $D$ except for a double pole at $z=0$, and such that

$$
f(z)= \begin{cases}\bar{z} & \text { on } \Gamma_{1} \\ \bar{z}+\lambda & \text { on } \Gamma_{2} \quad(\lambda \text { being some complex number }) .\end{cases}
$$

Then $D$ admits a q.i. (*) for every $h \in L_{a, s}^{1}(D)$.

Proof. Set $f(z)=a / z^{2}+b / z+T(z)$ where $T(z)$ is analytic in $D$. Let $h(z)$ be continuous in $\bar{D}$ and $h(z) \in L_{a, s}^{1}(D)$. This means that $\int_{\Gamma_{1}} h d z=\int_{\Gamma_{2}} h d z$ $=0$. Hence, using Green's formula,

\footnotetext{
${ }^{1}$ See, for instance, [3, p. 114], where this result is formulated (in much stronger form) for the $L^{2}(D)$-metric. The proof is based on the fact that any function in $L_{a}^{2}(G)$ ( $G$ is a simply connected bounded Jordan domain) can be approximated in the $L^{2}(D)$-metric by polynomials. But this last assertion is also true for the case of $L^{1}(D)$-approximation (see, for instance, [4, p. 45]). So the proof in [3] holds for our case as well.
} 


$$
\begin{aligned}
\int_{D} h d \sigma & =\frac{1}{2 i} \int_{C} \bar{z} h(z) d z=\frac{1}{2 i} \int_{\Gamma_{1}} f(z) h(z) d z+\frac{1}{2 i} \int_{\Gamma_{2}}[f(z)-\lambda] h(z) d z \\
& =\frac{1}{2 i} \int_{C} f(z) h(z) d z=\frac{1}{2 i} \int_{C}\left(\frac{a}{z^{2}}+\frac{b}{z}+T(z)\right) h(z) d z=A h^{\prime}(0)+B h(0)
\end{aligned}
$$

(where $A=\pi a, B=\pi b$ ). Thus, the q.i. holds for $h \in L_{a, s}^{1}(D)$ provided $h$ is continuous in $\bar{D}$. Let now $h(z)$ be any function in $L_{a, s}^{1}(D)$. In view of Lemma 1 , we can find a sequence $R_{n}(z)$ such that $\int_{D} h d \sigma=\lim _{n \rightarrow \infty} \int_{D} R_{n}(z) d \sigma$, each $R_{n}(z)$ having a single-valued integral in $D$.

Consequently we have

$$
\begin{aligned}
\int_{D} h(z) d \sigma & =\lim _{n \rightarrow \infty} \int_{D} R_{n}(z) d \sigma=\lim _{n \rightarrow \infty}\left(A R_{n}^{\prime}(0)+B R_{n}(0)\right) \\
& =A h^{\prime}(0)+B h(0)
\end{aligned}
$$

LEMMA 3. Let $\Delta$ be a closed annulus $1 \leqslant|z| \leqslant R$ and let $1<\alpha<R$. Then for every real $\lambda$ there exist a function $g(z)$ which is analytic in $\Delta$, and another function $f(z)$ which is analytic in $\Delta$ except for a double pole at $z=\alpha$, such that

$$
f(z)= \begin{cases}\bar{g}(z) & \text { on }|z|=1 \\ \bar{g}(z)+\lambda & \text { on }|z|=R\end{cases}
$$

Proof. Let $f(z)=a /(z-\alpha)^{2}+b /(z-\alpha)+T(z)$ where $a, b$ are real and $T(z)$ is analytic in $\Delta$. We then have the expansions for $f$ :

$$
\begin{aligned}
f(z)= & \frac{a}{\alpha^{2}} \sum_{0}^{\infty}(k+1)\left(\frac{z}{\alpha}\right)^{k}-\frac{b}{\alpha} \sum_{0}^{\infty}\left(\frac{z}{\alpha}\right)^{k} \\
& +\sum_{1}^{\infty} T_{k} z^{k}+\sum_{1}^{\infty} T_{-k} z^{-k}+T_{0} \quad \text { on }|z|=1, \\
f(z) & =\frac{a}{z^{2}} \sum_{0}^{\infty}(k+1)\left(\frac{\alpha}{z}\right)^{k}+\frac{b}{z} \sum_{0}^{\infty}\left(\frac{\alpha}{z}\right)^{k} \\
& +\sum_{1}^{\infty} T_{k} z^{k}+\sum_{1}^{\infty} T_{-k} z^{-k}+T_{0} \text { on }|z|=R .
\end{aligned}
$$

For $g(z)$ which is analytic in $\Delta$ we have

(4) $\overline{g(z)}+\lambda=\sum_{1}^{\infty} \bar{g}_{k} R^{2 k} z^{-k}+\sum_{1}^{\infty} \bar{g}_{-k} R^{-2 k} z^{k}+\bar{g}_{0}+\lambda$ on $|z|=R$.

Provided $f(z)=\overline{g(z)}$ on $|z|=1$, we obtain 


$$
\begin{aligned}
\frac{a}{\alpha^{2}}-\frac{b}{\alpha}+T_{0} & =\bar{g}_{0} ; \\
(k+1) \frac{a}{\alpha^{k+2}}-\frac{b}{\alpha^{k+1}}+T_{k} & =\bar{g}_{-k}, \quad T_{-k}=\bar{g}_{k} \quad(k=1,2, \ldots) .
\end{aligned}
$$

Provided $f(z)=\overline{g(z)}+\lambda$ on $|z|=R$, we obtain

$$
\begin{array}{r}
T_{0}=\bar{g}_{0}+\lambda, \quad(k-1) a \alpha^{k-2}+b \alpha^{k-1}+T_{-k}=\bar{g}_{k} R^{2 k}, \quad T_{k}=R^{-2 k} \bar{g}_{-k} \\
(k=1,2, \ldots) .
\end{array}
$$

From (5) and (6) we obtain

$$
\begin{gathered}
\lambda=b / \alpha-a / \alpha^{2}, \\
\text { (8) } \bar{g}_{k}=g_{k}=\frac{a(k-1) \alpha^{k-2}+b \alpha^{k-1}}{R^{2 k}-1}, \\
\bar{g}_{-k}=g_{-k}=\left(\frac{a}{\alpha^{k+2}}(k+1)-\frac{b}{\alpha^{k+1}}\right) \frac{R^{2 k}}{R^{2 k}-1} \quad(k=1,2, \ldots), \\
T_{0}=\bar{g}_{0}+\lambda, \\
\text { (9) } T_{k}=\left(\frac{a}{\alpha^{k+2}}(k+1)-\frac{b}{\alpha^{k+1}}\right) \frac{1}{R^{2 k}-1}, \quad T_{-k}=\frac{a(k-1) \alpha^{k-2}+b \alpha^{k-1}}{R^{2 k}-1} \\
(k=1,2, \ldots) .
\end{gathered}
$$

Using (8), (9) we obtain after a simple manipulation:

$$
\begin{aligned}
f(z)= & \frac{a}{(z-\alpha)^{2}}+\frac{b}{z-\alpha}+T_{0}+\lambda \sum_{k=1}^{\infty} \frac{(\alpha / z)^{k}-(\alpha / z)^{-k}}{R^{2 k}-1} \\
& +\frac{a}{\alpha^{2}} \sum_{k=1}^{\infty} k \frac{(\alpha / z)^{k}+(\alpha / z)^{-k}}{R^{2 k}-1} \\
& g(z)=\bar{T}_{0}-\lambda+\lambda \sum_{k=1}^{\infty} \frac{(\alpha z)^{k}-R^{2 k}(\alpha z)^{-k}}{R^{2 k}-1} \\
& +\frac{a}{\alpha^{2}} \sum_{k=1}^{\infty} \frac{(\alpha z)^{k}+R^{2 k}(\alpha z)^{-k}}{R^{2 k}-1} .
\end{aligned}
$$

We note, that for the present $T_{0}$ may be chosen arbitrarily; $a$ and $b$ may also be arbitrary (and real) but must satisfy (7). Since $1<\alpha<R, g(z)$ and the regular part of $f(z)$ are analytic in the closed annulus $\Delta$ and the above computation (5), (6) shows, that $f$ and $g$ satisfy the requirement of Lemma 3.

LEMMA 4. The function $g(z)$ which is defined by (11) is univalent in the closed annulus $\Delta$, provided $\alpha=R^{3 / 4}, a=\lambda \alpha^{2}(\lambda \neq 0)$, and provided $R$ is sufficiently large. 
Proof. Provided $\lambda=a / \alpha^{2}$, we obtain from (11): $g(z)=\bar{T}_{0}-\lambda+\lambda \phi(z)$, where we set

$$
\phi(z)=\sum_{1}^{\infty} \frac{(\alpha z)^{k}-R^{2 k}(\alpha z)^{-k}}{R^{2 k}-1}+\sum_{1}^{\infty} k \frac{(\alpha z)^{k}+R^{2 k}(\alpha z)^{-k}}{R^{2 k}-1} .
$$

Provided $\lambda \neq 0$, it suffices to prove univalence of $\phi(z)$. We obtain from (12):

$$
\begin{aligned}
\phi(z) & =\frac{2 \alpha z}{R^{2}-1}+\sum_{2}^{\infty} \frac{(\alpha z)^{k}-R^{2 k}(\alpha z)^{-k}}{R^{2 k}-1}+\sum_{2}^{\infty} k \frac{(\alpha z)^{k}+R^{2 k}(\alpha z)^{-k}}{R^{2 k}-1} \\
& =\frac{2 \alpha z}{R^{2}-1}+\phi_{1}(z)
\end{aligned}
$$

For any pair of points $z_{1}, z_{2}$ in $\Delta$ we have

$$
\phi\left(z_{2}\right)-\phi\left(z_{1}\right)=\int_{z_{1}}^{z_{2}} \phi^{\prime}(z) d z=\frac{2 \alpha}{R^{2}-1}\left(z_{2}-z_{1}\right)+\int_{z_{1}}^{z_{2}} \phi_{1}^{\prime}(z) d z .
$$

We can choose the path of integration from $z_{1}$ to $z_{2}$ in such a way that its length will not exceed $3\left|z_{1}-z_{2}\right|$. Thus, in order to prove univalence of $\phi(z)$ we have to show that

$$
\max _{z \in \Delta}\left|\phi_{1}^{\prime}(z)\right|<2 \alpha / 3\left(R^{2}-1\right)
$$

From (13) we obtain

$$
\left|\phi_{1}^{\prime}(z)\right| \leqslant \alpha\left\{\sum_{k=2}^{\infty} k \frac{|\alpha z|^{k-1}+R^{2 k}|\alpha z|^{-k-1}}{R^{2 k}-1}+\sum_{k=2}^{\infty} k^{2} \frac{|\alpha z|^{k-1}+R^{2 k}|\alpha z|^{-k-1}}{R^{2 k}-1}\right\}
$$

$$
\begin{aligned}
& <2 \alpha \sum_{k=2}^{\infty} k^{2} \frac{|\alpha z|^{k-1}+R^{2 k}|\alpha z|^{-k-1}}{R^{2 k}-1} \\
& <4 \alpha \sum_{k=2}^{\infty} k^{2} \frac{|\alpha z|^{k-1}+R^{2 k}|\alpha z|^{-k-1^{2}}}{R^{2 k}} \\
& =\frac{4 \alpha}{R^{2}} \sum_{2}^{\infty} k^{2}\left|\frac{\alpha z}{R^{2}}\right|^{k-1}+\frac{4 \alpha}{|\alpha z|^{2}} \sum_{2}^{\infty} k^{2} \frac{1}{|\alpha z|^{k-1}} .
\end{aligned}
$$

If we choose now $\alpha=R^{3 / 4}$, we obtain from (15):

$$
\left|\phi_{1}^{\prime}(z)\right| \leqslant 4 R^{-5 / 4} \sum_{2}^{\infty} k^{2}\left(R^{-1 / 4}\right)^{k-1}+4 R^{-3 / 4} \sum_{2}^{\infty} k^{2}\left(R^{-3 / 4}\right)^{k-1}<C R^{-3 / 2}
$$

for sufficiently large $R$.

The validity of (14) for $\alpha=R^{3 / 4}$ is now clear, which proves the lemma.

\footnotetext{
2 This step is correct provided $R>2^{1 / 4}$.
} 
3. We now proceed to prove our theorem. We choose some real $\lambda(\lambda \neq 0)$ and some sufficiently large $R$ (for which Lemma 4 is valid). Set $\alpha=R^{3 / 4}, a$ $=\alpha^{2} \cdot \lambda, b=2 \lambda \alpha$. The function $g(z)$ is then defined by (11) up to the additive constant $\overline{T_{0}}$, chosen such that $g\left(R^{3 / 4}\right)=0$. In view of Lemma $4, w=g(z)$ is analytic and univalent in the closed annulus $\Delta=\{1 \leqslant|z| \leqslant R\}$ and maps its interior onto the doubly connected domain $D$, which contains zero. Set $\tilde{f}(w)=f\left(g^{-1}(w)\right.$ ), where $f$ is defined by (10) (with $\lambda, R, \alpha, a, b, T_{0}$ as chosen above). $\tilde{f}(w)$ is single valued and analytic in $D$ except for a double pole at $w=0$. By virtue of Lemma 3 , we have

$$
\tilde{f}(w)= \begin{cases}\bar{w} & \text { on } \Gamma_{1}=\{w: w=g(z),|z|=1\}, \\ \bar{w}+\lambda & \text { on } \Gamma_{2}=\{w: w=g(z),|z|=R\} .\end{cases}
$$

Since $g(z)$ is analytic and univalent in the closed annulus, the curves $\Gamma_{1}$ and $\Gamma_{2}$ satisfy the topological conditions of Lemma 1 and, hence, by Lemma 2, the domain $D$ admits a quadratus identity $(*)$ with $z_{0}=0$.

ACKNowledgement. I am grateful to my colleague D. Aharonov for stimulating discussions and useful information.

\section{REFERENCES}

1. D. Aharonov and H. Shapiro, Domains on which analytic functions satisfy quadrature identities, J. Analyse Math. (to appear).

2. _ A minimal area problem in conformal mapping, Royal Inst. Tech. Res. Bull., 1973, 34 pp.

3. S. N. Mergelyan, On the completeness of systems of analytic functions, Uspehi Mat. Nauk 8 (1953), no. 4 (56), 3-63; English transl., Amer. Math. Soc. Transl. (2) 19 (1962), 109-166. MR 15, $411 ; 24$ \# A1410.

4. J. L. Walsh, Interpolation and approximation by rational functions in the complex domain, 2nd ed., Amer. Math. Soc. Colloq. Publ., vol. 20, Amer. Math. Soc., Providence, R.I., 1956.

Department of Mathematics, Technion, IsRael Institute of Technology, Haifa, Israel 